\title{
Expanding Plasma Region of an Inductively Driven Hydrogen Discharge
}

\author{
Zhivko Kiss'ovski, Stanimir Kolev, Antonia Shivarova, and Tsanko Tsankov
}

\begin{abstract}
The spatial distribution of the plasma parameterselectron density and temperature as well as plasma and floating potentials-in the region of plasma expansion of an inductively driven low-pressure discharge in hydrogen is studied by probe diagnostics. Electron cooling and a decrease of the electron density with formation of regions with different axial gradients are outlined as basic features of the expanding plasmas. Thermal conductivity and electron energy flux from the driver are considered to be responsible for the plasma behavior at its expansion. The role of the thermal diffusion in the formation of the axial profile of the plasma density is also shown.
\end{abstract}

Index Terms-Hydrogen discharges, inductive discharges, lowpressure discharges, plasma expansion, probe diagnostics.

\section{INTRODUCTION}

$\mathbf{S}$ TUDIES ON plasma expansion regions in different types of plasma sources (helicon sources [1]-[9], electron cyclotron resonance plasma reactors [10]-[12], and inductively coupled plasmas [13]-[18]) based on low-pressure discharges form an area of increasing recent and current activity. The construction of the plasma sources used in the plasma processing technology [19] basically involving a process chamber with expanding plasmas motivates such studies in discharges in different types of gases (Ar [1], [3], [5], [6], [8]-[12], [14]-[18], $\mathrm{He}$ [2], [13], $\mathrm{H}_{2}$ [4], [7], [20], $\mathrm{N}_{2}$ [11], $\mathrm{O}_{2}$ [13], $\mathrm{SF}_{6}$ [9], and $\mathrm{Ar}: \mathrm{SF}_{6}$ [14] and $\mathrm{He}-\mathrm{SiH}_{4}$ [13] mixtures). In particular, interest in studying hydrogen discharges is initiated also by their application as ion beam sources [21]-[24] of the neutral beam injectors of fusion machines. However, understanding the mechanisms that ensure plasma existence and determine the plasma behavior in expansion volumes is, no doubt, a complex problem that is attractive also for basic research.

Certainly, the process of plasma expansion depends on many parameters, such as the geometry and dimensions of both the driver and the expansion chamber, gas pressure, gas flow, RF power applied for the discharge production, and magnetic fields. This leads to complicated discharge behavior. For example, the pressure range between parts of militorrs and tens of militorrs appears to be split into two regions with different types of behavior of the axial profiles of the plasma parameters [5], [16].

Manuscript received August 7, 2006; revised March 30, 2007. This work was supported in part by the EURATOM Project FU06-CT-2003-00139, by Project F-1316 of the National Science Fund in Bulgaria, and by DFG Project 436 BUL 113/144/0-1.

The authors are with the Faculty of Physics, Sofia University, BG-1164 Sofia, Bulgaria (e-mail: ashiva@phys.uni-sofia.bg).

Digital Object Identifier 10.1109/TPS.2007.901938
Results for a rapid decrease of the plasma potential at the transition from the driver to the expansion-plasma volume and, respectively, registration of hot ions accelerated in the potential drop obtained at about and below 1 mtorr call for mechanisms of plasma expansion based on double-layer formation [5], [7], [9], [16], [25]-[29]. The current-free double layers discovered in this pressure range attract attention that motivates active research, both experimental and theoretical, on the conditions of their existence. Since the appearance of the double layers correlates with a large mean free path of the ions, compared to the characteristic lengths of the spatial variations of the $\mathrm{dc}$ potential, of the plasma density, and of the magnetic field (in discharges in an external magnetic field), threshold values with respect to the gas pressure (and to the magnetic field) as well as the influence of the type of the gas have been discussed. Due to the many factors determining the double-layer formation, they do not always appear [1], [12], even when the pressure is within the limits of (0.2-2) mtorr, which is usually considered [29] as a gas-pressure range of their existence.

The absence of abrupt jumps of the dc potential and, respectively, of double layers when the gas pressure is more than 3 mtorr has created the view [5] that the plasma expansion in this pressure range, when an external magnetic field is not applied to the expanding plasma volume, is well understood: The ion distribution is isotropic, and the electron density, as a whole, is considered to obey Boltzmann's law [1], [5], [12]. Complicated ion dynamics and ion temperature anisotropy (higher perpendicular ion temperature than the parallel one, with both being defined with respect to the magnetic field) arise when the plasma expands into a volume with a strongenough external magnetic field [8], [30]-[32]. In addition, results from recent experiments [17] on plasma expansion in an inductively driven source in argon gas show that the higher pressure range-about $10 \mathrm{mtorr}$ - possesses a peculiar behavior even when an external magnetic field is not applied to the plasma expansion volume: The electron temperature drops fast away from the driver, and regions of different rates of the axial decrease of the plasma density are formed in the expanding plasma volume. This brings up the question of whether such a behavior is a general characteristic - for discharges in different gases - of the expanding plasmas when the gas pressure is in the range of tens of militorrs.

The results presented here are from probe diagnostics of the expansion-plasma region of an inductively driven discharge in hydrogen. The gas-pressure range covered in the experiment is $p=(10-50)$ mtorr. The applied RF power (at $27 \mathrm{MHz}$ ) is the other external parameter that is varied. Both radial and 


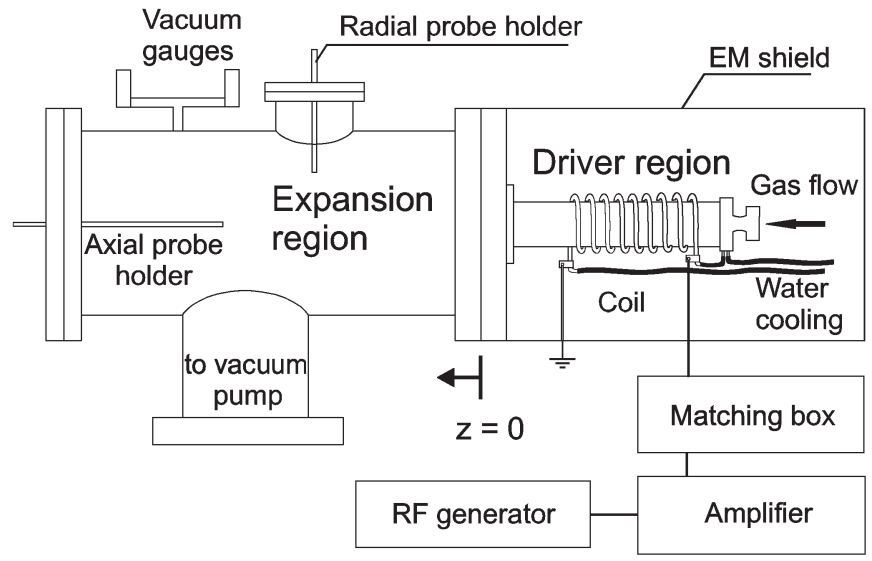

Fig. 1. Experimental setup.

axial variations of the plasma parameters have been studied by using two movable Langmuir probes. The difficulties in obtaining the plasma parameters from probe characteristics that were recorded from hydrogen discharges stem from the complexity of the ion composition in the discharge (three types of positive ions $\mathrm{H}^{+}, \mathrm{H}_{2}^{+}$, and $\mathrm{H}_{3}^{+}$as well as negative ions $\mathrm{H}^{-}$). In this connection, the electron density is determined from the electron energy distribution function (EEDF) and not from the ion saturation current of the probe characteristics, as has been done before [16], [17] in argon discharges. Spatial variation of the plasma parameters-electron temperature and density as well as plasma and floating potentials-completes the set of obtained results. The discussion is concentrated mainly on the axial decrease of the electron density and temperature showing formation of regions with different axial gradients in hydrogen discharges, as has been observed before in discharges in argon gas. Estimations based on solutions of the electron energy balance and of the momentum equation of the electrons show that: 1) nonlocal heating due to the electron energy flux from the driver is responsible for the formation of the axial profile of the electron temperature and, in general, for the behavior of the expanding plasmas in the pressure range considered and 2) the axial decrease of the electron temperature drives the mechanism of thermal diffusion, which determines the experimentally obtained structuring of the axial profile of the plasma density and its strong deviation from the Boltzmann's law relation.

\section{Experimental Setup And Diagnostic Procedure}

The experimental setup (Fig. 1) is the same as has been described before [17], [18]: an inductively driven plasma source with two parts of the discharge vessel. The active part of the discharge (its driver region) is a cylindrical type of inductive discharge produced by the RF current in a coil positioned around a quartz tube (with internal and external diameters $d_{1}=4.5 \mathrm{~cm}$ and $d_{2}=4.9 \mathrm{~cm}$, respectively, and a length of $l=30 \mathrm{~cm})$. A stainless steel cylinder $\left(D_{1}=22 \mathrm{~cm}\right.$ and $D_{2}=23 \mathrm{~cm}$ are its internal and external diameters, respectively, and $L=47 \mathrm{~cm}$ is the length) connected to the tube provides the volume for plasma expansion from the driver. The $\mathrm{RF}$ power input to the coil (a nine-turn water-cooled copper tube) is from an ICOM IC-718 RF generator and ACOM 2000A linear amplifier, through a matching box. The discharge is in hydrogen gas at pressure $p=(10-50)$ mtorr. The RF power sustaining the discharge is at $27 \mathrm{MHz}$ and varied up to $700 \mathrm{~W}$; the reflected power (deduced from the forward power) is less than $10 \%$.

The measurements are carried out in the volume of the expanding plasma by using two movable probes: 1) a probe positioned at the axis of the chamber (the end of the quartz tube is the zero axial position in the presentation of the results) and 2) a radial probe positioned at $z=12 \mathrm{~cm}$. The cylindrical probe tips are tungsten wires with a diameter of $0.5 \mathrm{~mm}$ and lengths of 5.6 and $5.8 \mathrm{~mm}$, respectively, of the axial and radial probes. The nonactive parts of the probe wires are protected from the plasma by ceramic tubes. Although the driver region has not been studied, the probe design is according to the requirements [33] for probe diagnostics of RF discharges: The passive compensation [34], [35] used for avoiding the RF distortions of the probe characteristics is implemented by choke filters, at the fundamental frequency and its second harmonics, and floating electrodes that are connected through capacitors to the probes. The data acquisition system [36] for recording the probe characteristics includes a ramp generator (at a frequency of $130 \mathrm{~Hz}$ ) for biasing the probe (with respect to the grounded chamber), a sensing resistor for the probe current measurements, a digital two-channel oscilloscope HP5410B, and a personal computer. The probe characteristics for each position of the probes is obtained after averaging over 256 traces.

The computer processing of the probe characteristics $(800$ data points per characteristics) includes [36] the following: 1) numerical smoothing of the characteristics by SavitzkyGolay filter and 2) numerical differentiation for obtaining the second derivative of the probe current (three-point differentiation with an adaptive step is applied).

The numerical code for calculation of the plasma parameters provides results for the following: 1) the plasma potential obtained from the crossing point of the second derivative of the probe current with the abscissa (the voltage axis); 2) the EEDF $F(\varepsilon)$ obtained from the Druyvesteyn formula [37]; 3 ) the electron density determined after integration of $F(\varepsilon)$; and 4) the electron temperature obtained from the slope of the semilogarithmic plot of $F_{p}(\varepsilon)=F(\varepsilon) / \sqrt{\varepsilon}$, which is considered as more reliable-compared to integration over $F(\varepsilon)$-when the EEDF is close to a Maxwellian one. Fig. 2 shows that, under the conditions of the experiment presented here, the body of the EEDF, excluding its high-energy tail, is very well approximated by a Maxwellian distribution. In addition, the directed velocity of the plasma flow is very low, i.e., smaller than both the thermal electron velocity and the ion sound velocity, thus not influencing the applicability of the probe diagnostics procedure.

\section{Spatial Distribution of the Plasma PARAMETERS: RESULTS AND DISCUSSIONS}

The obtained results are for the spatial distribution of the plasma parameters-electron density and temperature as well as floating and plasma potentials-in the expanding plasma volume of the source operation in a hydrogen gas. The structure 


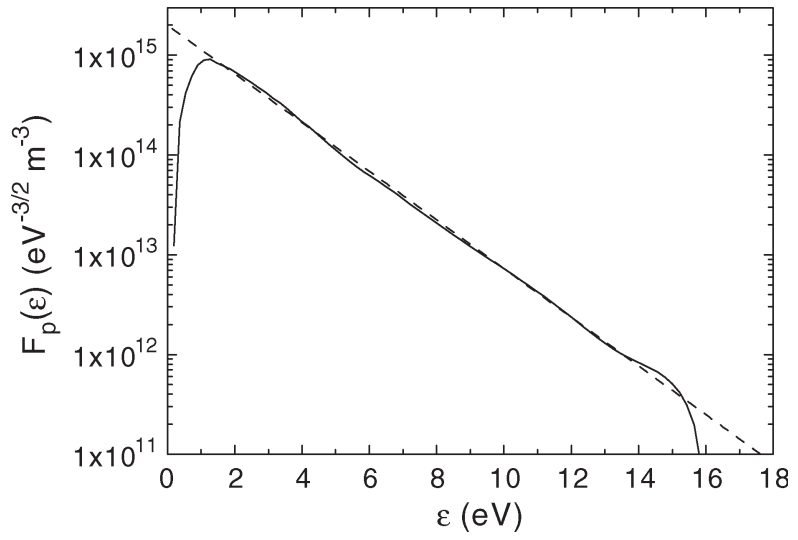

Fig. 2. Example of measured $F_{p}(\varepsilon)=F(\varepsilon) / \sqrt{\varepsilon}$ (solid curve) and its approximation (dashed curve) by a Maxwellian distribution.

of the discharge is presented by axial and radial profiles of the plasma parameters for three values of the gas pressure $p=14,26$, and 40 mtorr and of the applied RF power $P=$ 300,500 , and $700 \mathrm{~W}$.

\section{A. Axial Profiles of the Plasma Parameters}

The axial structure of the expanding plasma region is presented by the results for the axial variation of the electron density (Fig. 3) and temperature (Fig. 4), and of the plasma (Fig. 5) and floating (Fig. 6) potentials measured at the axis of the metallic chamber.

Electron density $n_{e}$ decreases away from the driver (Fig. 3), however, with changing gradient along the length of the expanding plasma volume. Three regions complete the axial profile of the density: 1) a fast drop of $n_{e}$ in the region $(z \leq 2 \mathrm{~cm})$ close to the driver; 2) a plateau region for $2 \mathrm{~cm} \leq z \leq 6 \mathrm{~cm}$; and 3) a slow decrease for $z \geq 6 \mathrm{~cm}$. Similar structuring of the axial profile of the electron density has also been observed before in argon discharges [16], [17]. Increasing applied power leads to a density increase by keeping the trends of the axial variation. The plateau region of the $n_{e}(z)$ profile is better pronounced when the applied power is higher. The pressure dependence is more complicated. For a given applied power, the electron density close to the driver is higher when the gas pressure is higher, and the axial density gradient for $z \geq$ $6 \mathrm{~cm}$ is also higher. The former is in accordance [38], [39] with the expectation for the pressure dependence of $n_{e}$ in the driver: lower losses due to charged particle fluxes to the walls for higher pressure, resulting in higher density for a constant applied power. However, this is not the case in the region away from the driver, e.g., at $z \geq 12 \mathrm{~cm}$, the electron density is lower when the gas pressure is higher. This could be associated with a strong coupling of the expansion-plasma region with the driver and, thus, an importance of the charged particle flux from the driver for the plasma existence in the expanding plasma volume: A lower charged particle flux from the driver for higher gas pressure should result in a lower plasma density, as the measurements show. Charge-exchange collisions limiting the ion motion from the driver is excluded as an explanation for the reduced electron density at higher neutral pressure since the threshold value of the ion energy [40] for collisions of $\mathrm{H}_{3}^{+}$
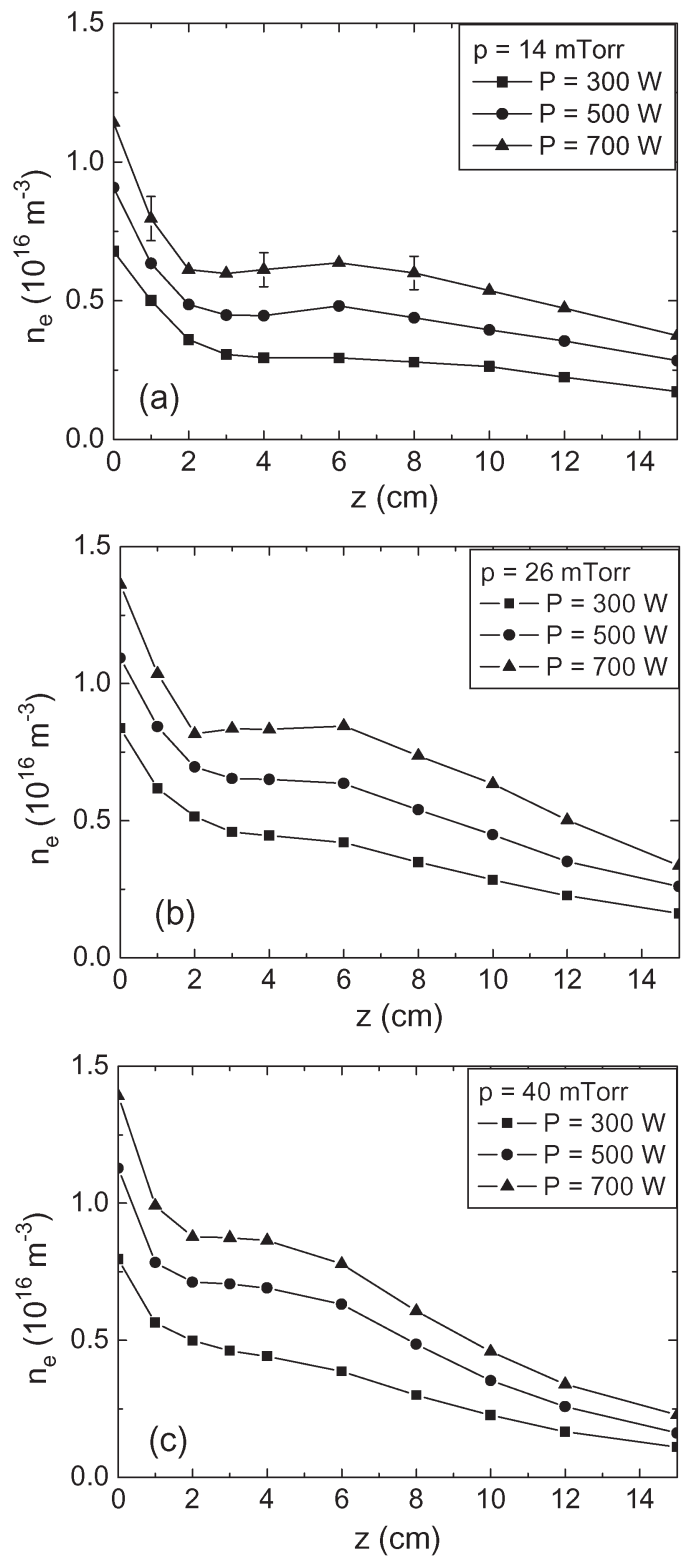

Fig. 3. Axial profiles of the electron density for different values of the applied RF power (as marked on the figures) at the three values of gas pressure: (a) $p=14$ mtorr, (b) 26 mtorr, and (c) 40 mtorr.

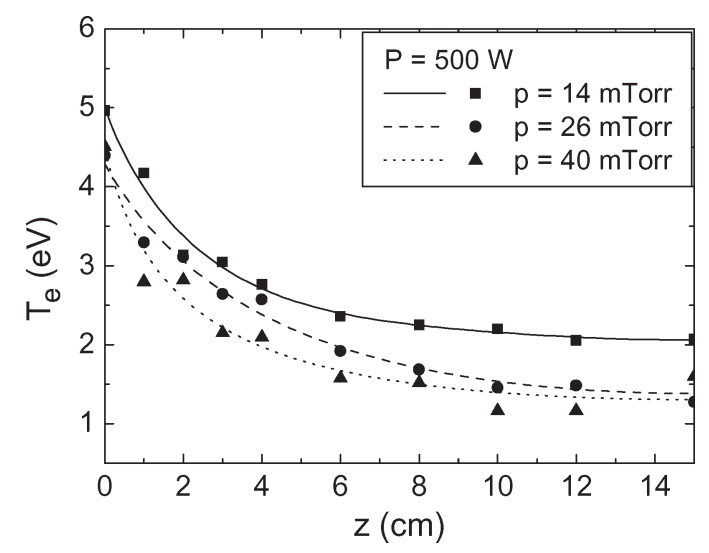

Fig. 4. Axial profiles of the electron temperature for the three values of gas pressure ( $p=14,26$, and 40 mtorr) and $P=500 \mathrm{~W}$. Experimental data are presented by symbols and fitting curves. 


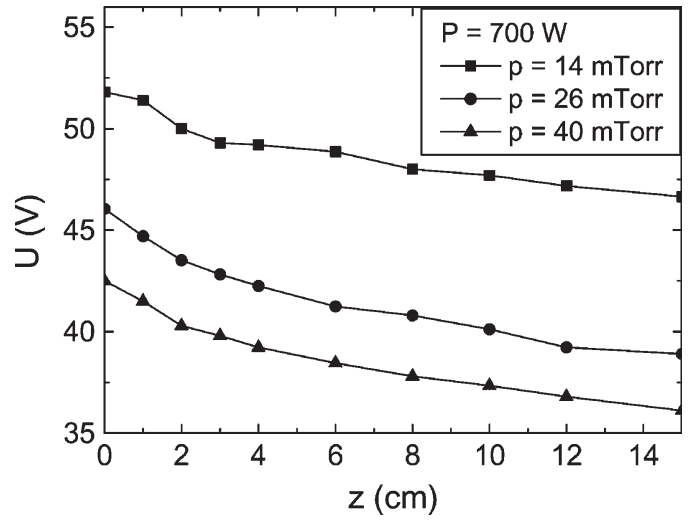

Fig. 5. Axial profiles of the plasma potential for the three values of gas pressure ( $p=14,26$, and $40 \mathrm{mtorr})$ and $P=700 \mathrm{~W}$.

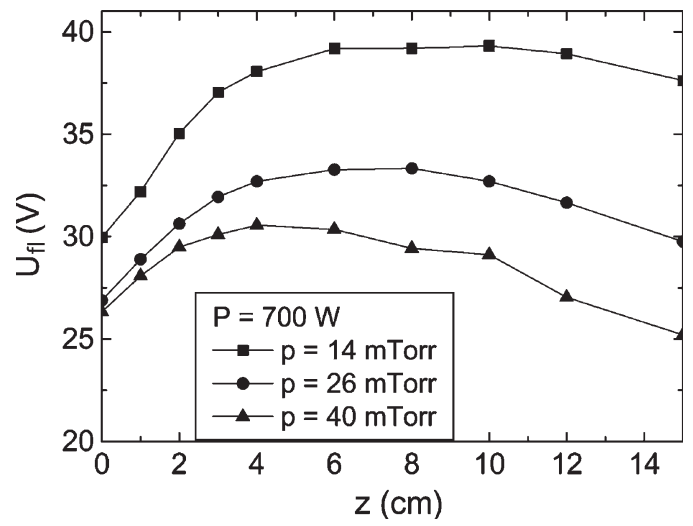

Fig. 6. Same as in Fig. 5 but for the floating potential $U_{\mathrm{fl}}$.

ions with hydrogen molecules $\mathrm{H}_{2}$ is high and not possible to be reached in the dc field measured (Fig. 5). $\mathrm{H}_{3}^{+}$and $\mathrm{H}_{2}$ are involved in the estimations of the charge-exchange collisions since, in the range of the measured values of the electron density, these are the ion and neutral species with the highest densities [41].

The axial decrease of the electron density is accompanied by an axial decrease of the electron temperature $T_{e}$ (Fig. 4). However, here, the regions of different rates of axial variation are two: 1) a region of a faster drop of $T_{e}$ close to the driver $(z \leq 6 \mathrm{~cm})$, which covers the first two regions of the $z$-variation of $n_{e}$ (the fast drop of $n_{e}$ and the $n_{e}(z)$-plateau region), and 2 ) a slower decrease of $T_{e}$ away from the driver $(z \geq 6 \mathrm{~cm})$. The electron temperature increases with the decrease of the gas pressure, and it does not depend on the applied RF power, both in accordance with the general trends of the gas-discharge behavior. The values of the electron temperature at $z=0$ are in agreement with the results for the electron temperature from the model [42] of low-pressure hydrogen discharges.

Plasma potential $U$ (Fig. 5) decreases along the axis of the expanding plasma region, in accordance with the axial variation of $T_{e}$. The axial electric field is of the order of that registered before [5], [12] in expanding plasmas without double-layer formation in argon discharges. The axial variation of the floating potential $U_{\mathrm{fl}}$ (Fig. 6) is nonmonotonic. With the obtained weaker axial variation of $U$ compared with that of $T_{e}$, the nonmonotonic axial changes of $U_{\mathrm{fl}}$ are in accordance with

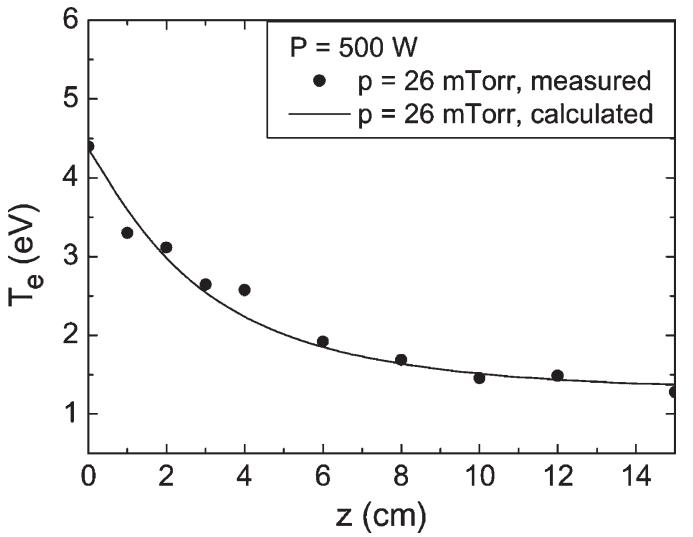

Fig. 7. Comparison of the experimental data (by symbols) for the axial decrease of the electron temperature for $p=26$ mtorr $(P=500 \mathrm{~W})$ with the theoretical result (solid curve) for the axial profile of $T_{e}$ obtained as a solution of (1).

the well-known relation $U_{\mathrm{fl}}=U-\left(\kappa T_{e} / e\right) \ln \left(\sqrt{m_{i} / m_{e}}\right)$ between $U_{\mathrm{fl}}$ and $U$, which was checked to be fulfilled for the experimental data in Figs. 4-6; here, $\kappa$ is the Boltzmann's constant, $e$ is the electron charge, and $m_{e}$ and $m_{i}$ are the masses of electrons and ions, respectively. The comparatively weak axial changes of the plasma potential is also an indication of the strong coupling between the driver and the expansion-plasma volume that was already mentioned regarding the axial profile of the electron density.

Electron cooling at the plasma expansion (Fig. 4) is the main conclusion from the experiment that was also made before but for argon discharges [17]. The very weak axial variation of $T_{e}$ away from the driver calls for the importance of nonlocality that is associated with thermal conductivity effects. This is confirmed by Fig. 7 where experimental data for $T_{e}(z)$ are compared with an axial profile of the electron temperature obtained by solving (in cylindrical coordinates $r, \varphi$, and $z$, and with an azimuthal symmetry) the equation

$$
\frac{1}{r} \frac{\partial}{\partial r}\left(r \frac{\partial \Psi}{\partial r}\right)+\frac{\partial^{2} \Psi}{\partial z^{2}}=0
$$

for the potential

$$
\Psi\left(T_{e}\right)=\int_{0}^{T_{e}} \chi_{e}\left(T_{e}^{\prime}\right) d T_{e}^{\prime}
$$

of the thermal flux

$$
J_{e}=-\chi_{e} \nabla T_{e}
$$

of the electrons. Here, $\chi_{e}=(5 / 2) n_{e} D_{e}$, with $D_{e}$ being the diffusion coefficient of the electrons, is the thermal conductivity coefficient. In obtaining the solution $\Psi(r, z)$ of (1), the spatial variation of the plasma density is disregarded. The values of $\Psi(r, z)$ at the boundaries $z=0, z=L$, and $r=R=D_{1} / 2$ are calculated from the experimental results for $T_{e}$ according to the relation $\Psi\left(T_{e}\right)=(\beta / 2) T_{e}^{2}$ between $T_{e}$ and $\Psi\left(T_{e}\right)$ obtained from (2); $\beta=(5 / 2)\left(n_{e} / m_{e} \nu_{e-n}\right)$ and $\nu_{e-n}=\nu_{e-H}+\nu_{e-H_{2}}$ is the electron-neutral elastic collision frequency. The very 


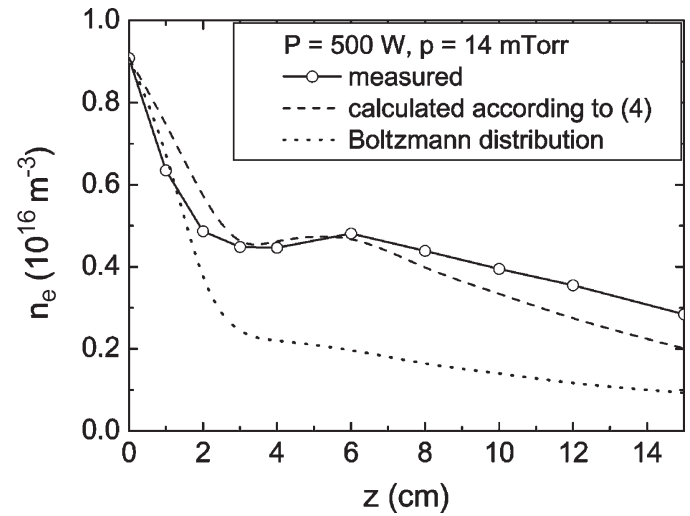

Fig. 8. Comparison of the experimental data (symbols and solid curve) for the axial variation of the electron density for $p=14 \operatorname{mtorr}(P=500 \mathrm{~W})$ with the theoretical result [(4) (dashed curve)]. The Boltzmann's law distribution (6) also shown (dotted curve) is taken with the averaged value of $T_{e}=2.9 \mathrm{eV}$ obtained under the given gas-discharge conditions in the expanding plasma region.

good agreement between the experimental and theoretical results for the axial profile of $T_{e}$ shows that the electron energy input carried by the particle flux from the driver and the spatial distribution of this energy input through thermal conductivity are the main mechanisms ensuring plasma maintenance in the expansion volume.

The structuring of the axial profile of the electron density, with a formation of a plateau region between the regions of the fast drop of $n_{e}$ close to the driver and the slow decrease away from it, is the other conclusion from the experiment. Such a behavior of the axial variation of $n_{e}$ is in agreement (Fig. 8) with the solution

$$
n_{e}(z)=n_{e}(z=0) \frac{T_{e}(z=0)}{T_{e}(z)} \exp \left(\int_{0}^{z} \frac{e}{\kappa T_{e}\left(z^{\prime}\right)} \frac{d U}{d z^{\prime}} d z^{\prime}\right)
$$

of the momentum equation of the electrons

$$
e n_{e} \frac{d U}{d z}-\frac{d}{d z}\left(n_{e} \kappa T_{e}\right)=0
$$

obtained by accounting not only for the axial changes of the potential $U$ but also for the axial variation of $T_{e}$. In plotting (4) in Fig. 8, the experimental data for the $U(z)$ and $T_{e}(z)$ dependences have been employed. An assumption for a constant value of the electron temperature reduces (4) to the Boltzmann's law relation

$$
n_{e}(z)=n_{e}(z=0) \exp \left\{\frac{e}{\kappa T_{e}}[U(z)-U(z=0)]\right\}
$$

between $n_{e}(z)$ and $U(z)$ that was considered before [1], [5], [12] as a characteristic of the expanding plasmas in the pressure range above a few millitorrs. The obtained (Fig. 8) strong deviation from the Boltzmann's law and the good agreement of the experimental results for $n_{e}(z)$ with (4) show the important role of the thermal diffusion-disregarded in the derivation of (6) - in the formation of the axial profile of $n_{e}$.
Thus, the axial drop of $T_{e}$ (Figs. 4 and 7) at the plasma expansion strongly affects the axial variation of the plasma density, through the mechanism of the thermal diffusion.

The preceding estimations are in the trends of those made before [33], [43] regarding experiments, which were in the same gas-pressure range, on plasma expansion-however, in a straight tube with the same diameter as of the driver region-from a helicon discharge in argon gas. The case of the inductive driving of the discharge, for a zero magnetic field, has also been considered. Comparison of the results presented here with the experimental results and the corresponding estimations in [33] and [43] shows differences that could be attributed to: 1) different ranges of variation of plasma density and, more importantly, 2) different sizes of the expanding plasma volumes (respectively, the radius of the expanding plasma region that is quite larger than that of the driver, as stated here, and the radius of the expanding plasma region that is equal to that of the driver, as in [33] and [43]). In both cases, electron cooling and thermal conductivity effects appear to be responsible for the axial variation of the electron temperature in the expanding plasma volume. However, whereas the high electron density in the experiments [33], [43] in helicon discharges drives Spitzer-Härm type of heat flux [44], involving electron-electron collisions in the thermal conductivity coefficient, the low electron density in the experiment presented here determines the importance of the classical thermal conductivity [38] governed by the elastic electron-neutral collision frequency. The shapes of the axial profiles of electron temperature and electron density obtained before [33], [43] and here are essentially different, and this may be attributed to the different sizes of the expanding plasma regions. The plasma expansion in a straight tube, as shown in the inductive mode operation of a helicon discharge, results in an abrupt drop of the electron temperature and a monotonic decrease of the electron density, followed by a long-length region of almost constant $T_{e}$ and $n_{e}$. Compared to these results, the axial profile of $T_{e}$ (Fig. 4) obtained for plasma expansion in a larger radius volume (than that of the driver) is smoother, and the axial profile of $n_{e}$ has a complicated behavior. The different trends of the axial profiles of the plasma parameters obtained here could be related to the smaller radial losses of charged particles when the transverse size of the expanding plasma volume exceeds considerably the radius of the driver. However, the explanation of the mechanisms of the plasma expansion in the two cases (of different sizes of expanding plasma volumes) needs intensive theoretical work based on development of 2-D models of the discharges.

\section{B. Radial Profiles of the Plasma Parameters}

The radial profiles of the plasma parameters are measured at a distance that is comparatively far from the driver, i.e., at $z=12 \mathrm{~cm}$. The radial changes of the electron density and temperature shown in Fig. 9 are for varying applied power, at a given gas pressure. The electron temperature is not influenced by the applied power, and it has almost constant value over the cross section, with a slight increase toward the walls. The latter case may be attributed to secondary electrons accelerated in the high potential drop near the walls, influencing the plasma 


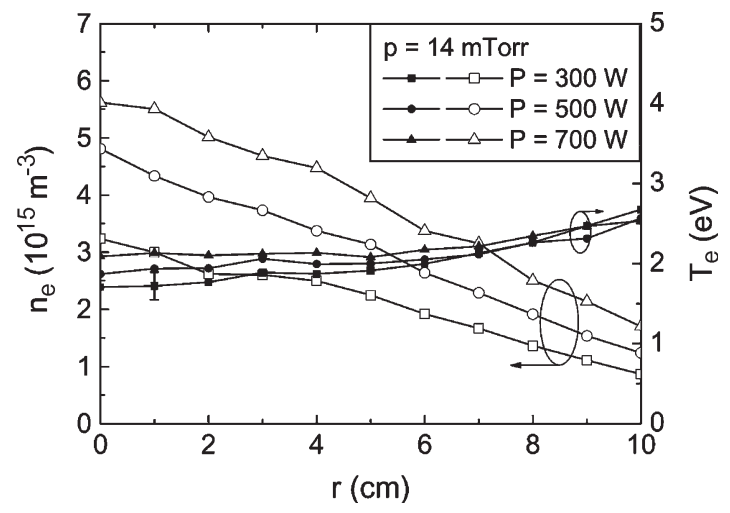

Fig. 9. Radial profiles of the electron density (open symbols) and temperature (closed symbols) measured at $z=12 \mathrm{~cm}$, with $p$ and $P$ values as marked on the figure.

characteristics there. The average value of $n_{e}$ increases with the applied power. The results from measurements at different values of the gas pressure show the decrease of the plasma density with the gas pressure increase, already commented in the discussion on the axial profiles of $n_{e}$.

\section{CONCLUSION}

The results on the radial and axial distribution of the plasma parameters in the expansion volume of an inductively coupled plasma source operating in hydrogen, which are presented here, reveal the spatial structure of the discharge. The low-pressure discharges that are studied are sustained in the tens-of-millitorr range. The spatial variation of the electron temperature shows the cooling of the plasma due to its expansion in a larger volume. The axial decrease of the electron temperature is accompanied with complicated structuring of the axial variation of the electron density. Although a strong decrease of the electron temperature is shown, the values obtained are not low enough for ensuring optimum conditions for the volume production of negative hydrogen ions via dissociative attachment of electrons to vibrationally excited hydrogen molecules. This confirms the necessity of additional cooling of the electrons by magnetic filters [22], even in the ion beam sources with expanding plasma regions.

The similarity of the axial profiles of the electron temperature and density in hydrogen discharges with those in discharges in argon gas shows that general mechanisms, and not a strong influence of the nature of the given gas, are responsible for forming the main trends of the spatial structure of the expanding plasmas in the pressure range above a few militorrs. The simple approach to the axial profile of the electron temperature shown here based on the electron energy flux gives the indication that the electron energy input from the driver combined with thermal conductivity effects is the right direction in outlining the mechanisms of plasma existence in the expansion volume. This means a strong coupling of the plasma expansion region to the driver. Moreover, with the axial changes of the electron temperature and the electron cooling at the plasma expansion, the thermal diffusion becomes important, causing strong deviations from the Boltzmann's law distribution of the electron density and leading to complicated structuring of its axial profile.

\section{REFERENCES}

[1] C. Charles, R. W. Boswell, and R. K. Porteous, "Measurements and modelling of ion energy distribution functions in a low pressure argon plasma diffusing from a $13.56 \mathrm{MHz}$ helicon source," J. Vac. Sci. Technol. A, Vac. Surf. Films, vol. 10, no. 2, pp. 398-403, Mar. 1992.

[2] R. H. Goulding, D. Pavarin, F. Angrilli, G. C. Barber, M. D. Carter, R. Maggiora, and D. O. Sparks, "Helicon plasma source configuration analysis by means of density measurements," in Proc. ICEAA, Torino, Italy, 1999, pp. 107-110.

[3] F. F. Chen, X. Jiang, and J. D. Evans, "Plasma injection with helicon sources," J. Vac. Sci. Technol. A, Vac. Surf. Films, vol. 18, no. 5, pp. 2108 2115, Sep. 2000.

[4] M. D. Carter, F. W. Baity, Jr., G. C. Barber, R. H. Goulding, Y. Mori, D. O. Sparks, K. F. White, E. F. Jaeger, F. R. Chang-Diaz, and J. P. Squire, "Comparing experiments with modeling for light ion helicon plasma sources," Phys. Plasmas, vol. 9, no. 12, pp. 5097-5110, Dec. 2002.

[5] C. Charles and R. Boswell, "Current-free double-layer formation in a high-density helicon discharge," Appl. Phys. Lett., vol. 82, no. 9, pp. 1356-1358, Mar. 2003.

[6] R. L. Kinder, A. R. Ellingboe, and M. J. Kushner, "H- to W-mode transitions and properties of a multimode helicon plasma reactor," Plasma Sources Sci. Technol., vol. 12, no. 4, pp. 561-575, Nov. 2003.

[7] C. Charles, "Hydrogen ion beam generated by a current-free double layer in a helicon plasma," Appl. Phys. Lett., vol. 84, no. 3, pp. 332-334, Jan. 2004.

[8] X. Sun, C. Biloiu, R. Hardin, and E. E. Scime, "Parallel velocity and temperature of argon ions in an expanding, helicon source driven plasma," Plasma Sources Sci. Technol., vol. 13, no. 3, pp. 359-370, Aug. 2004.

[9] X. Sun, A. M. Keesee, C. Biloiu, and E. E. Scime, "Observation of ionbeam formation in a current-free double layer," Phys. Rev. Lett., vol. 95, no. 2, pp. $025004.1-025004.4$, Jul. 2005.

[10] D. J. Trevor, N. Sadeghi, T. Nakano, J. Derouard, R. A. Gottscho, P. D. Foo, and J. M. Cook, "Spatially resolved ion velocity distributions in a diverging field electron cyclotron resonance plasma reactor," Appl. Phys. Lett., vol. 57, no. 12, pp. 1188-1190, Sep. 1990.

[11] Y. Weng and M. J. Kushner, "Electron energy distributions in electron cyclotron resonance discharges for materials processing," J. Appl. Phys., vol. 72, no. 1, pp. 33-42, Jul. 1992.

[12] A. Aanesland and C. Charles, "Plasma expansion from a dielectric electron cyclotron resonance source," Phys. Scr., vol. T122, pp. 19-24, 2006.

[13] I. Pérès and M. J. Kushner, "Spatial distributions of power and ion densities in RF excited remote plasma reactors," Plasma Sources Sci. Technol., vol. 5, no. 3, pp. 499-509, Aug. 1996.

[14] N. Plihon, C. S. Corr, P. Chabert, and J.-L. Raimbault, "Periodic formation and propagation of double layers in the expanding chamber of an inductive discharge operating in $\mathrm{Ar} / \mathrm{SF}_{6}$ mixtures," J. Appl. Phys., vol. 98, no. 2, pp. $023306.1-023306.7$, Jul. 2005.

[15] H. J. Yoon, C. Charles, and R. W. Boswell, "A comparison between experimental results and a fluid description of a low pressure discharge driven by a double-saddle antenna," J. Phys. D: Appl. Phys., vol. 38, no. 16, pp. 2825-2829, Aug. 2005.

[16] V. N. Volynets, W. Park, Yu. N. Tolmachev, V. G. Pashkovsky, and J. Yoo, "Spatial variation of plasma parameters and ion acceleration in an inductive plasma system," J. Appl. Phys., vol. 99, no. 4, pp. $043302.1-$ 043302.5 , Feb. 2006.

[17] M. Dimitrova, N. Djermanova, Zh. Kiss'ovski, S. Kolev, A. Shivarova, and Ts. Tsankov, "Probe diagnostics of expanding plasmas at low gas pressure," Plasma Process. Polym., vol. 3, no. 2, pp. 156-161, 2006.

[18] Ts. Tsankov, Zh. Kiss'ovski, N. Djermanova, and S. Kolev, "Electron energy distribution function measurements in an inductively driven tandem plasma source," Plasma Process. Polym., vol. 3, no. 2, pp. 151-155, 2006.

[19] J. R. Roth, Industrial Plasma Engineering. Bristol, PA: Inst. Phys. Publ., 1995.

[20] G. J. H. Brussaard, M. van der Steen, M. Carrère, M. C. M. van de Sanden, and D. C. Schram, "Langmuir probe measurements in an expanding magnetized plasma," Phys. Rev. E, Stat. Phys. Plasmas Fluids Relat. Interdiscip. Top., vol. 54, no. 2, pp. 1906-1911, Aug. 1996.

[21] H. W. Loeb, "Plasma-based ion beam sources," Plasma Phys. Control. Fusion, vol. 47, no. 12B, pp. B565-B576, Dec. 2005.

[22] E. Speth, H. D. Falter, P. Franzen et al., "Overview of the RF source development programme at IPP Garching," Nucl. Fusion, vol. 46, no. 6 , pp. S220-S238, Jun. 2006.

[23] A. G. Nikitin, F. El Balghiti, and M. Bacal, "Comparison of negative ion density measurements by probes and by photodetachment," Plasma Sources Sci. Technol., vol. 5, no. 1, pp. 37-42, Feb. 1996. 
[24] M. B. Hopkins, M. Bacal, and W. G. Graham, "Electron energy distribution functions and negative ion concentrations in tandem and hybrid multicusp negative hydrogen ion sources," J. Phys. D: Appl. Phys., vol. 24, no. 3, pp. 268-276, Mar. 1991.

[25] T. Sato and H. Okuda, "Ion-acoustic double layers," Phys. Rev. Lett., vol. 44, no. 11, pp. 740-743, Mar. 1980.

[26] F. W. Perkins and Y. C. Sun, "Double layer without current," Phys. Rev. Lett., vol. 46, no. 2, pp. 115-118, Jan. 1981.

[27] D. L. Newman, M. V. Goldman, R. E. Ergun, and A. Mangeney, "Formation of double layers and electron holes in a current-driven space plasma," Phys. Rev. Lett., vol. 87, no. 25, pp. 255 001.1-255 001.4, Nov. 2001.

[28] N. Plihon, C. S. Corr, and P. Chabert, "Double layer formation in the expanding region of an inductively coupled electronegative plasma," Appl. Phys. Lett., vol. 86, no. 9, pp. 091 501.1-091 501.3, Feb. 2005.

[29] M. A. Lieberman, C. Charles, and R. W. Boswell, "A theory for formation of a low pressure, current-free double layer," J. Phys. D: Appl. Phys., vol. 39, no. 15, pp. 3294-3304, Aug. 2006.

[30] E. E. Scime, P. A. Keiter, M. W. Zintl, M. M. Balkey, J. L. Kline, and M. E. Koepke, "Control of ion temperature anisotropy in a helicon plasma," Plasma Sources Sci. Technol., vol. 7, no. 2, pp. 186-191, May 1998.

[31] J. L. Kline, M. M. Balkey, P. A. Keiter et al., "Ion dynamics in helicon sources," Phys. Plasmas, vol. 10, no. 5, pp. 1-9, May 2003.

[32] E. E. Scime, P. A. Keiter, M. M. Balkey, R. F. Boivin, J. L. Kline, M. Blackburn, and S. P. Gary, "Ion temperature anisotropy limitation in high beta plasmas," Phys. Plasmas, vol. 7, no. 5, pp. 2157-2165, May 2000.

[33] I. D. Sudit and F. F. Chen, "Discharge equilibrium of a helicon plasma," Plasma Sources Sci. Technol., vol. 5, no. 1, pp. 43-53, Feb. 1996.

[34] R. R. J. Gagné and A. Cantin, "Investigation of an RF plasma with symmetrical and asymmetrical electrostatic probes," J. Appl. Phys., vol. 43, no. 6, pp. 2639-2646, Jun. 1972.

[35] I. D. Sudit and F. F. Chen, "RF compensated probes for high-density discharges," Plasma Sources Sci. Technol., vol. 3, no. 2, pp. 162-168, May 1994

[36] N. Djermanova, Zh. Kiss'ovski, and Ts. Tsankov, "EEDF determination by numerical processing of the probe data in neon DC discharge," Contrib. Plasma Phys., vol. 46, no. 4, pp. 312-321, 2006.

[37] M. J. Druyvesteyn, "Der niedervoltbogen,” Z. Phys., vol. 64, pp. 781-798, 1930.

[38] V. E. Golant, A. P. Zhilinsky, and I. E. Sakharov, Fundamentals of Plasma Physics. New York: Wiley, 1980.

[39] Yu. P. Raizer, Gas Duscharge Physics. Berlin, Germany: SpringerVerlag, 1991.

[40] A. V. Phelps, "Cross sections and swarm coefficients for $\mathrm{H}^{+}, \mathrm{H}_{2}^{+}, \mathrm{H}_{3}^{+}$, $\mathrm{H}, \mathrm{H}_{2}$, and $\mathrm{H}^{-}$in $\mathrm{H}_{2}$ for energies from $0.1 \mathrm{eV}$ to $10 \mathrm{keV}$," J. Phys. Chem. Reference Data, vol. 19, no. 3, pp. 653-675, May 1990.

[41] Ts. Paunska, H. Schlüter, A. Shivarova, and Kh. Tarnev, "Surface-wave produced discharges in hydrogen-II: Modification of the discharge structure for varying gas-discharge conditions," Plasma Sources Sci. Technol., vol. 12 , no. 4, pp. 608-618, Nov. 2003.

[42] Ts. Paunska, H. Schlüter, A. Shivarova, and Kh. Tarnev, "Low pressure hydrogen discharges," Phys. Plasmas, vol. 13, no. 2, pp. 023 504.1023 504.10, Feb. 2006.

[43] F. F. Chen, I. D. Sudit, and M. Light, "Downstream physics of the helicon discharge," Plasma Sources Sci. Technol., vol. 5, no. 2, pp. 173-180, May 1996.

[44] L. Spitzer, Jr. and R. Härm, "Transport phenomena in a completely ionized gas," Phys. Rev., vol. 89, no. 5, pp. 977-981, Mar. 1953.

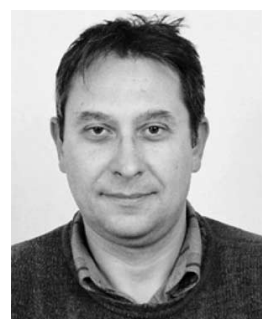

Zhivko Kiss'ovski received the M.Sc. and Ph.D. degrees in physics from Sofia University, Sofia, Bulgaria, in 1985 and 1996, respectively.

Since 1991, he has been with the Faculty of Physics, Sofia University, as a Physicist and an Assistant Professor, and has been an Associate Professor since 2006. His research interests are plasma physics (plasma diagnostics methods, waves, and instabilities in low-temperature magnetized plasmas) and gas discharges, i.e., dc discharges and highfrequency discharges (travelling-wave-sustained discharges and inductive discharges)

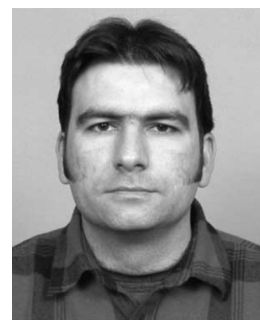

Stanimir Kolev received the M.Sc. and Ph.D degrees in physics from Sofia University, Sofia, Bulgaria, in 2001 and 2007, respectively. His Ph.D. thesis is on modeling and diagnostics of inductive discharges.

He is currently with the Faculty of Physics, Sofia University.

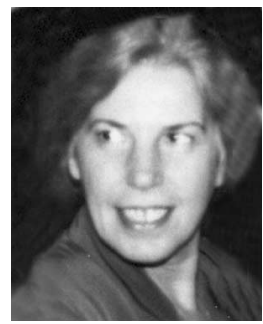

Antonia Shivarova received the M.Sc. and Ph.D degrees in physics from Sofia University, Sofia, Bulgaria, in 1970 and 1976, respectively, and the D.Sc. degree in physics in 1999.

Since 1976, she has been with the Faculty of Physics, Sofia University, where she was an Assistant Professor from 1976 to 1986 and an Associate Professor from 1986 to 2000, and has been a Full Professor since 2000. Her research interests are plasma physics (waves and instabilities, nonlinear wave interactions and nonlinear waves, and diagnostics), high-frequency gas discharges (travelling-wave-sustained discharges and inductive discharges) and, until recently, nonlinear optics (temporal and spatial solitons)

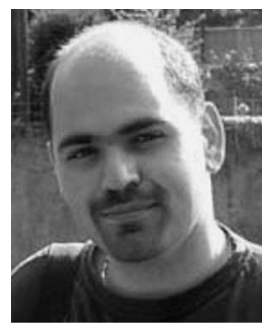

Tsanko Tsankov received the B.Sc. and M.Sc degrees in physics from Sofia University, Sofia, Bulgaria, in 2004 and 2005, respectively. $\mathrm{He}$ is currently working toward the Ph.D. degree in the Faculty of Physics, Sofia University. His thesis is on inductively driven negative hydrogen ionbeam sources, including both experimental and theoretical work. 